\title{
Segmented Optimal Multi-Degree Reduction Approximation of Bézier Curve
}

\author{
Zhi Wu \\ College of Mathematics \\ Inner Mongolia University for Nationalities \\ Tongliao 028043, China \\ E-mail: zhiwu0103@163.com \\ Yuedao Jiang \\ College of Mathematics \\ Inner Mongolia University for Nationalities \\ Tongliao 028043, China \\ E-mail: jiangyuedaogaojing@163.com \\ Genzhu Bai (Corresponding author) \\ College of Mathematics \\ Inner Mongolia University for Nationalities \\ Tongliao 028043, China \\ E-mail: bgz049@163.com
}

Received: November 11, 2011

Accepted: December 5, 2011

Published: January 1, 2012

doi:10.5539/cis.v5n1p49

URL: http://dx.doi.org/10.5539/cis.v5n1p49

\begin{abstract}
This paper presents a segmented optimal multi-degree reduction approximation method for Bézier curve based on the combination of optimal function approximation and segmentation algorithm. In the proposed method, each Bernstein basis function is optimally approximated by the linear combination of lower power $\mathrm{S}$ bases. The piecewise curve of Bernstein basis function is replaced by the obtained optimal approximation functions. The proposed method is simple and intuitive. Experiments manifest that it improves the approximation performance.
\end{abstract}

Keywords: S power base, Linear normed space, Optimal approximation, Subdivision

\section{Introduction}

Approximating the high-degree polynomal curves and surfaces with the low-degree ones is one of the important research topics in Computer Aided Design (CAD). In geometrical modelling tasks, it also plays an important role in data compression, data transfer and data exchange. In recent years, many works on Bézier curve approximation with degree reduction have been reported. For instance, Sánchez-Reyes proposes an approximation algorithm. In this algorithm, Bernstein base function is first represented by $\mathrm{S}$ power bases, and then the high-degree terms in the new curve equation expressed by $\mathrm{S}$ power bases are removed (Sánchez-Reyes, 1997, p.319-357). Hoschek proposes to first divide the original curve into segments and then acquires the interpolation approximation based on multiple low-degree curves (Hoschek, 1987, p.59-66). Bogacki proposes a method to transform the base function. Based on this method, the multi-degree reduction approxiamation is achieved (Bogacki, 1995, p.651-661). The method of inverse-degree-ascension is also proposed (Piegl, 1995, p.101-110) for degree reduction. Cheng manifests that low-degree curve can be acquired based on the geometry information of the control points (Cheng, 1997, p.55-58).

The shape of Bézier curve is controlled by the geometric clues conveyed by its control points. Therefore, the shape of Bézier curve would be changed if the corresponding geometric clues are revised. The degree reduction 
of Bézier curve should not only preserve the shape of the original polynomal curves, but also should minimize the approximation error. To avoid the effect of the control points to the shape of the curve, we propose to reduce the degree of Bézier function. Therefore, we transform the Bézier curve degree reduction into the problem of function approximation. It is intuitive to infer that, once the optimal approximating elements for a certain function are found, the corresponding optimal approximation curve could be acquired.

Based on the work of Zhang (Zhang, 2008, p.80-85), we further propose a segmented optimal multi-degree reduction approximation method for Bézier curve. This method is built on function approximation theory. According to this theory, a banach space is constructed by the meaning of two-norm. In this banach space, the optimal approximating elements expressed by the linear combinations of lower S power bases can be found for the given high-degree Bernstein base functions. Then, the segmented optimal multi-degree reduction approximation of Bézier curve can be acquired based on the linear combination of the control points obtained by the segmentation algorithm and the obtained optimal approximating elements.

\section{The Theoretical Problem}

In the normed space, the research on approximation theory is focused on two aspects: approximating a function in a certain function space with the functions from its subspaces, and constructing the optimal approximation according to the given criteria.

\section{Proposition 2.1}

Suppose $K$ is a number field, the set $X=\left\{0, \pm t^{i}\right\}_{i=0}^{n}(t \in[0,1])$ is a linear space in the $K$.

It is easy to prove that the Proposition 2.1 is true.

\section{Definition 2.2}

Suppose $X=\left\{0, \pm t^{i}\right\}_{i=0}^{n}(t \in[0,1])$ is the linear space in the number filed $K$, we define the function $\|\bullet\|: X \rightarrow R^{1}$, any $u \in X$, where

$$
\|u\|=\left(\int_{[0,1]}|u(t)|^{2} d t\right)^{1 / 2} .
$$

\section{Proposition 2.3}

$(X,\|\bullet\|)$ is the linear normed space in the number field $K$.

Proof: For any $u \in X,\|u\|=\left(\int_{[0,1]}|u(t)|^{2} d t\right)^{1 / 2} \geq 0 \quad$ (Positive definite).

For any $u, v \in X,\|u+v\|=\left(\int_{[0,1]}|u(t)+v(t)|^{2} d t\right)^{1 / 2}$,

According to the Minkowski inequality, i.e.,

$$
\|u+v\|=\left(\int_{[0,1]}|u(t)+v(t)|^{2} d t\right)^{1 / 2} \leq\left(\int_{[0,1]}|u(t)|^{2} d t\right)^{1 / 2}+\left(\int_{[0,1]}|v(t)|^{2} d t\right)^{1 / 2} .
$$

For any $u \in X$ and any $a \in K$, we can infer that,

$$
\|a u\|=\left(\int_{[0,1]}|a u(t)|^{2} d t\right)^{1 / 2}=|a|\left(\int_{[0,1]}|u(t)|^{2} d t\right)^{1 / 2} \text { (Homogeneity). }
$$

Therefore, the linea space $X$ is the banach space.

Theorem 2.4 (Wang, 2001, chapter2)

(The theorem regrading the existence of the optimal approximation) Suppose $M$ is a finite dimensional subspace in the normed space $X$, then for each $x \in X$, there exists an optimal approximation of $x$ in $M$.

Theorem 2.5 (Wang, 2001, chapter2)

Suppose $X$ is a strictly convex normed space, and the subspace $M \subset X$, then the maximum number of the optimal approximation of any $x \in X$ in $M$ is 1 .

\section{Degree Reduction Method of Optimal Approximation}

Definition 3.1 (Sánchez-Reyes, 1997, p.319-357)

Given an integer $p \geq 1$, we define the following base function as the P-order S Power base function: 


$$
\left\{s^{k}(1-t), s^{k} t, s^{p}\right\}_{i=0}^{p-1} \cup\left\{s^{k}(1-t), s^{k} t,\right\}_{i=0}^{p-1}, s=(1-t) t, t \in[0,1] .
$$

According to the above definition, if $n \geq 2 p$, then

$$
\left(\left\{s^{k}(1-t), s^{k} t, s^{k} t, s^{p}\right\}_{i=0}^{p-1} \cup\left\{s^{k}(1-t), s^{k} t, s^{k}\right\}_{i=0}^{p-1}\right) \subset\left\{0, \pm t^{i}\right\}_{i=0}^{n}, t \in[0,1] .
$$

Suppose $n \geq 2 p$, for each Bernstein base function, we reduce its degree and find its approximation in the following ways:

Suppose $n$ is odd and the reduced degree is odd, suppose $n$ is odd and the reduced degree is even

$$
\begin{aligned}
& \min \left\|\frac{B_{i}^{n}(t)}{C_{i}^{n}}-\sum_{k=0}^{p-1}\left(a_{k}^{i} s^{k}(1-t)+b_{k}^{i} s^{k} t\right)-c_{k}^{i} s^{p}\right\|^{2} \\
& \text { s.t. } \quad 1-\sum_{k=0}^{p-1}\left(a_{k}^{i} s^{k}(1-t)+b_{k}^{i} s^{k} t\right)-c_{k}^{i} s^{p} \geq 0 \\
& \sum_{k=0}^{p-1}\left(a_{k}^{i} s^{k}(1-t)+b_{k}^{i} s^{k} t\right)-c_{k}^{i} s^{p} \geq 0 \\
& i=0,1, \cdots, n \\
& \min \left\|\frac{B_{i}^{n}(t)}{C_{i}^{n}}-\sum_{k=0}^{p-1}\left(a_{k}^{i} s^{k}(1-t)+b_{k}^{i} s^{k} t\right)\right\|^{2} \\
& \text { s.t. } \quad 1-\sum_{k=0}^{p-1}\left(a_{k}^{i} s^{k}(1-t)+b_{k}^{i} s^{k} t\right)-c_{k}^{i} s^{p} \geq 0 \\
& \sum_{k=0}^{p-1}\left(a_{k}^{i} s^{k}(1-t)+b_{k}^{i} s^{k} t\right)-c_{k}^{i} s^{p} \geq 0 \\
& i=0,1, \cdots, n
\end{aligned}
$$

Suppose $n$ is even and the reduced degree is odd, suppose $n$ is even and the reduced degree is even

$$
\begin{aligned}
& \min \left\|\frac{B_{i}^{n}(t)}{C_{i}^{n}}-\sum_{k=0}^{p-1}\left(a_{k}^{i} s^{k}(1-t)+b_{k}^{i} s^{k} t\right)\right\|^{2} \\
& \text { s.t. } \quad 1-\sum_{k=0}^{p-1}\left(a_{k}^{i} s^{k}(1-t)+b_{k}^{i} s^{k} t\right)-c_{k}^{i} s^{p} \geq 0 \\
& \sum_{k=0}^{p-1}\left(a_{k}^{i} s^{k}(1-t)+b_{k}^{i} s^{k} t\right)-c_{k}^{i} s^{p} \geq 0 \\
& i=0,1, \cdots, n \\
& \min \left\|\frac{B_{i}^{n}(t)}{C_{i}^{n}}-\sum_{k=0}^{p-1}\left(a_{k}^{i} s^{k}(1-t)+b_{k}^{i} s^{k} t\right)-c_{k}^{i} s^{p}\right\|^{2} \\
& \text { s.t. } \quad 1-\sum_{k=0}^{p-1}\left(a_{k}^{i} s^{k}(1-t)+b_{k}^{i} s^{k} t\right)-c_{k}^{i} s^{p} \geq 0 \\
& \sum_{k=0}^{p-1}\left(a_{k}^{i} s^{k}(1-t)+b_{k}^{i} s^{k} t\right)-c_{k}^{i} s^{p} \geq 0 \\
& i=0,1, \cdots, n
\end{aligned}
$$

The two inequalities in the constraints restrict that the best approximating function falls within the range of the Bernstein base function with no coefficient.

Because of $B_{i}^{n}(1-t)=B_{i}^{n-i}(t)$, the optimal approximating function of $B_{i}^{n}(1-t)$ is 


$$
\sum_{k=0}^{p-1}\left(a_{k}^{i} s^{k}+b_{k}^{i} s^{k}(1-t)\right)
$$

and the optimal approximating function of $B_{i}^{n-i}(t)$ is

$$
\sum_{k=0}^{p-1}\left(a_{k}^{n-i} S^{k}(1-t)+b_{k}^{n-i} S^{k}\right)
$$

Then we can infer that

$$
\sum_{k=0}^{p-1}\left(a_{k}^{i} s^{k}+b_{k}^{i} s^{k}(1-t)\right)=\sum_{k=0}^{p-1}\left(a_{k}^{n-i} s^{k}(1-t)+b_{k}^{n-i} s^{k}\right) .
$$

According to the coefficient comparison, we get

$$
a_{k}^{i}=b_{k}^{n-i}, b_{k}^{i}=a_{k}^{n-i} .
$$

We have to satisfy the equality between the value of the Bernstein base function with no coefficient and the value of the optimal approximating function on the vertexes in the domain of definition, i.e.,

$$
\left\{\begin{array}{l}
\frac{B_{0}^{n}(0)}{C_{0}^{n}}=\frac{B_{n}^{n}(1)}{C_{n}^{n}}=a_{0}^{n}=b_{0}^{n}=1, \frac{B_{0}^{n}(1)}{C_{0}^{n}}=\frac{B_{n}^{n}(0)}{C_{n}^{n}}=a_{0}^{n}=b_{0}^{n}=0 \\
\frac{B_{i}^{n}(0)}{C_{i}^{n}}=\frac{B_{n-i}^{n}(1)}{C_{n-i}^{n}}=a_{0}^{i}=b_{0}^{n-i}=0, \frac{B_{i}^{n}(1)}{C_{i}^{n}}=\frac{B_{n-i}^{n}(0)}{C_{n-i}^{n}}=b_{0}^{i}=a_{0}^{n-i}=0 \\
1 \leq i \leq n-1 .
\end{array}\right.
$$

Where $a_{k}^{i}, b_{k}^{i}$ and $a_{k}^{n-i}, b_{k}^{n-i}$ denote the coefficients of the optimal approximating functions of $\frac{B_{i}^{n}(t)}{C_{i}^{n}}, \frac{B_{i}^{n-i}(t)}{C_{i}^{n-i}}$, respectively.

According to Eq. (1) and Eq. (2), we can get $n+1$ optimal approximating functions by computing the coefficients, i.e.,

$$
\left\{\begin{array}{l}
a_{k}^{i}, b_{k}^{i} \\
a_{k}^{i}, b_{k}^{i}, c_{p}^{p} \\
0 \leq i \leq\lfloor n / 2\rfloor, 1 \leq k \leq p .
\end{array}\right.
$$

Segmentation algorithm 3.2 (Zhang, 2008, p.80-85)

Suppose $A_{n}(t)$ is a $n$-degree Bézier curve, whose control vertexes are $p_{i}(i=0,1, \cdots, n)$

$$
A_{n}(t)=\sum_{i=0}^{n} p_{i} B_{i}^{n}(t) \quad t \in[0,1] .
$$

The segmentation of n-degree Bézier curve means equivalently dividing a Bézier curve into two sections, i.e.,

$$
A_{L}(u)=\sum_{i=0}^{n} p_{i}{ }^{(L)} B_{i}{ }^{n}(u) \text { and } A_{R}(u)=\sum_{i=0}^{n} p_{i}{ }^{(R)} B_{i}{ }^{n}(u) .
$$

Where

$$
A(t)=\left\{\begin{array}{ll}
A_{L}\left(\frac{t}{\lambda}\right) & 0 \leq t \leq \lambda \\
A_{R}\left(\frac{t-\lambda}{1-\lambda}\right) & \lambda \leq t \leq 1
\end{array} \quad(0 \leq \lambda \leq 1) .\right.
$$

The relationships between the control points of segmentation curve and original curve can be represented as: 


$$
p_{i}^{(L)}=\sum_{j=0}^{i} p_{j} B_{j}^{n}(\lambda), p_{i}^{(R)}=\sum_{j=i}^{n} p_{j} B_{j}^{n-i}(\lambda), i=0,1, \cdots, n .
$$

There are the segmentation curves of the segmented optimal multi-degree reduction approximation for the n-degree Bézier curve.

$$
\begin{aligned}
& A_{n-m}^{(L)}(t)=\sum_{i=0}^{n}\left[\sum_{k=0}^{p-1} p_{i}^{(L)} C_{i}^{n}\left(a_{k}^{i} s^{k}(1-t)+b_{k}^{i} s^{k} t\right)\right] \text { and } A_{n-m}^{(R)}(t)=\sum_{i=0}^{n}\left[\sum_{k=0}^{p-1} p_{i}^{(R)} C_{i}^{n}\left(a_{k}^{i} s^{k}(1-t)+b_{k}^{i} s^{k} t\right)\right] ; \\
& A_{n-m}^{(L)}(t)=\sum_{i=0}^{n} p_{i}^{(L)}\left[\sum_{k=0}^{p-1} C_{i}^{n}\left(d_{k} s^{k}(1-t)+b_{k}^{i} s^{k} t\right)+c_{p}^{i} s^{p}\right] \text { and } A_{n-m}^{R(R)}(t)=\sum_{i=0}^{n} p_{i}^{(R)}\left[\sum_{k=0}^{p-1} C_{i}^{n}\left(d_{k} s^{k}(1-t)+b_{k}^{i} s^{k} t\right)+c_{p}^{i} s^{p}\right] ;
\end{aligned}
$$

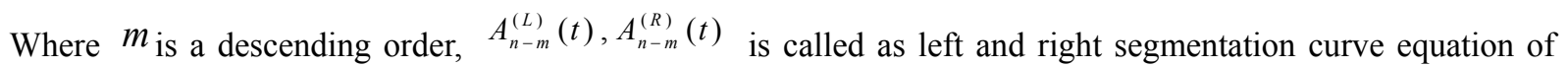
optimal approximation, respectively.

\section{Numerical Examples and the Error Comparison}

In this experiment, we compare the segmentation algorithm (Zhang, 2008, p.80-85) and our proposed method in nine-degree Bézier curve approximation. The parameter $\lambda$ of the Bézier curve is set as 0.5 and the control vertexes are set as $[0,0],[2,6],[3,6],[4,6],[6,2],[8,2],[9,5],[10,5],[11,4],[12,0]$.

The Bézier curve is denoted by solid line in Figure 1 and Figure 2. The approximation curves of the segmentation algorithm (Zhang, 2008, p.80-85) and our method are denoted by dashed lines in Figure 1 and Figure 2, respectively. The approximation errors of the two algorithms are compared in Table 1 . From the comparisons in Table 1, it is obvious that our proposed algorithm achieves more precise approximation than the segmentation algorithm. For instance on the vertex point with $\mathrm{x}=4$, the approximation error of our proposed algorithm is 173 times smaller than the one of the segmentation algorithm. This experiment clearly shows the advantage of our proposed method.

\section{Conclusions}

This paper proposes an optimal approximation method to the n-degree Bézier curve. The proposed method not only achieves small approximation error but also is intuitive and simple. In the further work, we will further take the non-negativity of the optimal approximating function into consideration. The precision of the low-degree approximation will be improved. Moreover, we will further study the scale of function approximation and the constraint condition.

\section{References}

Bogacki, P., Weinstein, S., \& Xu, Y. (1995). Degree Reduction of Bézier Curves by Uniform Approximation with Endpoint Interpolation. Computer Aided Geometric Design, 29(9), 651-661.

Cheng, Jiuping. (1997). Degree Reduction of Bézier Curves and Algorithm of Error Calculation. Journal of An Hui Industrial College, 16(4), 55-58. http://dx.doi.org/10.1016/0167-8396(87)90024-0

Hoschek, J. (1987). Approximation of Spline Curves. Computer Aided Geometric Design, 4(1), 59-66.

Piegl, L, \& Tiller, W. (1995). Algorithm for Degree Reduction of B-spline Curves. Computer Aided Geometric Design, 27(2), 101-110.

Sánchez-Reyes, J. (1997). The Symmetric Analogue of The Polynomial Power Basis. ACM Transactions on Graphics, 16(3), 319-357. http://dx.doi.org/10.1145/256157.256162

Wang, Changqing. (2001). Mathematical foundations of modern analytical applications. Xi'An: University of Electronic Science and Technology press, (Chapter 2).

Zhang, li, Tan, Jie-qing, \& Liu, zhi. (2008). Multi-Degree Reduction Approximation of Bézier Curves by S-Power Series Based on Subdivision. Journal of Engineering Graphics, 6(6), 80-85. 
Table 1. The Comparsion of Approximation Error

\begin{tabular}{|c|c|c|c|c|c|}
\hline \multirow{2}{*}{$\begin{array}{l}\text { Nine-degree } \\
\text { Bézier curve }\end{array}$} & $x$ & 2 & 4 & 8 & 10 \\
\hline & $y$ & 4.312270400 & 4.762363124 & 3.728230752 & 3.866363768 \\
\hline \multirow{3}{*}{$\begin{array}{l}\text { Approximation } \\
\text { curve of the } \\
\text { segmentation } \\
\text { algorithm (Zhang } \\
\text { li, 2008, p.80-85) }\end{array}$} & \multicolumn{3}{|c|}{ Left Segmentation Curve } & \multicolumn{2}{|c|}{ Right Segmentation Curve } \\
\hline & $x$ & 2 & 4 & 8 & 10 \\
\hline & $y_{1}$ & 4.198707699 & 4.630951747 & 3.711517830 & 3.770164075 \\
\hline $\begin{array}{l}\text { Approximation } \\
\text { Error }\end{array}$ & $\left|y-y_{1}\right|$ & 0.113562701 & $\mathbf{0 . 1 3 1 4 1 1 3 7 7}$ & 0.016712922 & 0.096199693 \\
\hline \multirow{3}{*}{$\begin{array}{l}\text { Approximation } \\
\text { curve of } \\
\text { our proposed } \\
\text { method }\end{array}$} & \multicolumn{3}{|c|}{ Left Segmentation Curve } & \multicolumn{2}{|c|}{ Right Segmentation Curve } \\
\hline & $x$ & 2 & 4 & 8 & 10 \\
\hline & $y_{2}$ & 4.318157926 & 4.761607212 & 3.729388636 & 3.863915914 \\
\hline $\begin{array}{l}\text { Approximatoin } \\
\text { Error }\end{array}$ & $\left|y-y_{2}\right|$ & 0.005887526 & 0.000755912 & 0.001157884 & 0.002447854 \\
\hline $\begin{array}{c}\text { The Comparsion } \\
\text { of Approximation } \\
\text { Error }\end{array}$ & $\frac{\left|y-y_{1}\right|}{\left|y-y_{2}\right|}$ & 19.28869630 & 173.84480865 & 14.434021024 & 39.299604061 \\
\hline
\end{tabular}

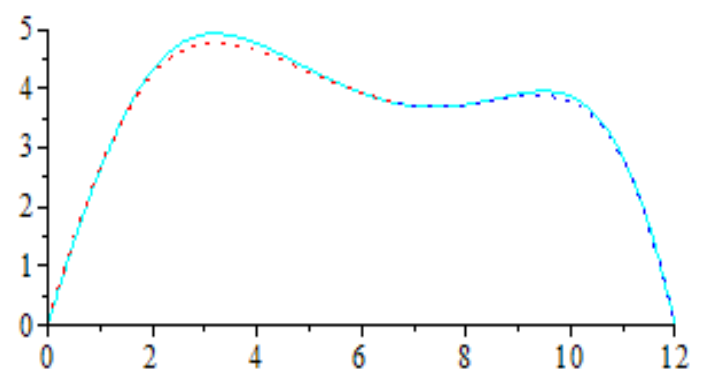

Figure 1. The approximation result of segmentation algorithm (Zhang li, 2008, p.80-85)

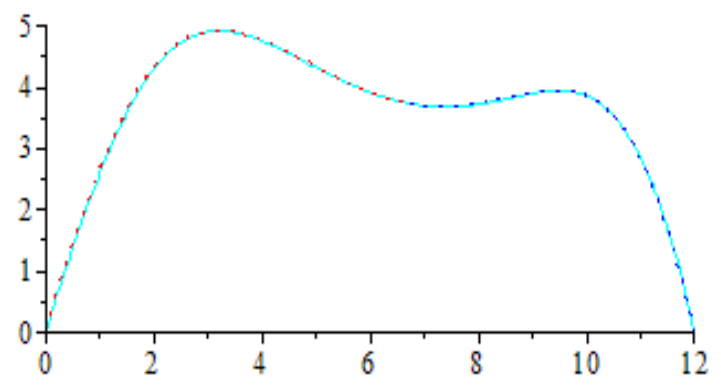

Figure 2. The approximation result of the proposed method 